\title{
Rocket nozzle cold-gas flow velocity measurements using laser-induced gratings
}

\author{
B. Hemmerling, ${ }^{1}$ M. Neracher, ${ }^{1 *}$ D. Kozlov, ${ }^{2}$ W. Kwan, ${ }^{3}$ R. Stark, ${ }^{3}$ D. Klimenko, ${ }^{3}$ W. Clauss ${ }^{3}$ \\ and $\mathrm{M}$. Oschwald ${ }^{3}$
}

${ }^{1}$ Paul Scherrer Institute, CH-5232 Villigen PSI, Switzerland

${ }^{2}$ General Physics Institute, RAS, 117991 GSP-1, Moscow, Russia

${ }^{3}$ DLR Lampoldshausen, German Aerospace Center, 74239 Hardthausen, Germany

Received 29 May 2002; Accepted 16 September 2002

\begin{abstract}
The heterodyne detection laser-induced electrostrictive gratings technique was employed for characterization of a flow velocities field in the plume of a rocket nozzle. The intention of the work was to validate CFD calculations, that predict the appearance of a recirculation zone inside the flow at a certain ratio of nozzle and ambient pressures. This zone co-exists with the side loads to the nozzle wall that were observed experimentally as transient forces during the start-up or the shut-down of the thrust chamber. Measurements were performed in cold-gas flows from a subscale thrust-optimized, parabolic nozzle. Nitrogen at stagnation pressures up to 40 bar was supplied to the nozzle. The experimental results clearly proved the existence of a recirculation zone in the flow field, in a reasonable agreement with the predictions of CFD calculations. Copyright $(2002$ John Wiley \& Sons, Ltd.
\end{abstract}

\section{INTRODUCTION}

The technique of laser-induced gratings (LIGs) has been demonstrated recently in a few laboratory measurements as a seedless method of non-intrusive instantaneous optical flow velocimetry. ${ }^{1-6}$ However, the applicability of laser-induced gratings to investigations of real fluid dynamics problems is still open to question. In the present work, the heterodyne detection laser-induced electrostrictive grating technique was used to validate computational fluid dynamics (CFD) calculations of the flow field in the plume of a subscale thrustoptimized, parabolic rocket nozzle. Investigations showed that at a certain ratio of nozzle wall pressure to ambient pressure the flow inside the nozzle separates from the wall, causing a system of shocks, which adapt the overexpanded flow to the ambient pressure. Two different flow patterns are possible, either when the separated flow continues as a free jet (free shock separation), or when the separated flow reattaches to the wall downstream of the separation point (restricted shock separation). ${ }^{7,8}$ The transition between these two flow patterns in thrust-optimized parabolic nozzles is accompanied by high lateral forces on the nozzle wall,

*Correspondence to: M. Neracher, Paul Scherrer Institute, CH-5232 Villigen PSI, Switzerland. E-mail: marc.neracher@psi.ch Contract/grant sponsor: Swiss Federal Department of Energy (BFE).

Contract/grant sponsor: Leonard Euler Centre of European Research Community on Flow, Turbulence and Combustion (LEC ERCOFTAC) Switzerland.

Contract/grant sponsor: Astrium GmbH. which, under unfavorable conditions, may cause damage to the nozzle. For the restricted shock separation pattern, CFD calculations predict an appearance of a recirculation zone in a certain location inside the flow. This recirculation zone is non-existent or is significantly smaller in the case of the free shock separation pattern.

The purpose of this work was to perform measurements of flow velocities in a few definite points inside the plume behind the nozzle and to verify, at certain stagnation pressures, the existence of the recirculation zone associated with the restricted shock separation inside the nozzle.

\section{LASER-INDUCED ELECTROSTRICTIVE GRATING TECHNIQUE}

Laser-induced gratings are spatially periodic modulations of material properties. They are generated as a response to the interference pattern formed by two short-pulse laser beams of the same wavelength $\lambda_{\mathrm{p}}$ and with wavevectors $\mathbf{k}_{1}$ and $\mathbf{k}_{2}$ intersecting at a shallow angle. The interaction area defines the sample volume [see Fig. 1(a)]. If the laser intensity is sufficiently high, two acoustic waves are generated by electrostriction. , $^{2,6}$ Electrostriction is a non-resonant effect, that takes place in gases of all polarizable molecules. The wavelength $\Lambda$ of these acoustic waves is defined by the fringe spacing of the interference pattern, they have equal amplitudes and propagate with adiabatic sound velocity $v_{\mathrm{s}}$ in the opposite directions, $\mathbf{q}$ and $-\mathbf{q}$, normally to the planes of the interference fringes. Here, $\mathbf{q}=\mathbf{k}_{\mathbf{2}}-\mathbf{k}_{\mathbf{1}}$ denotes the grating vector, $q=2 \pi / \Lambda$. 

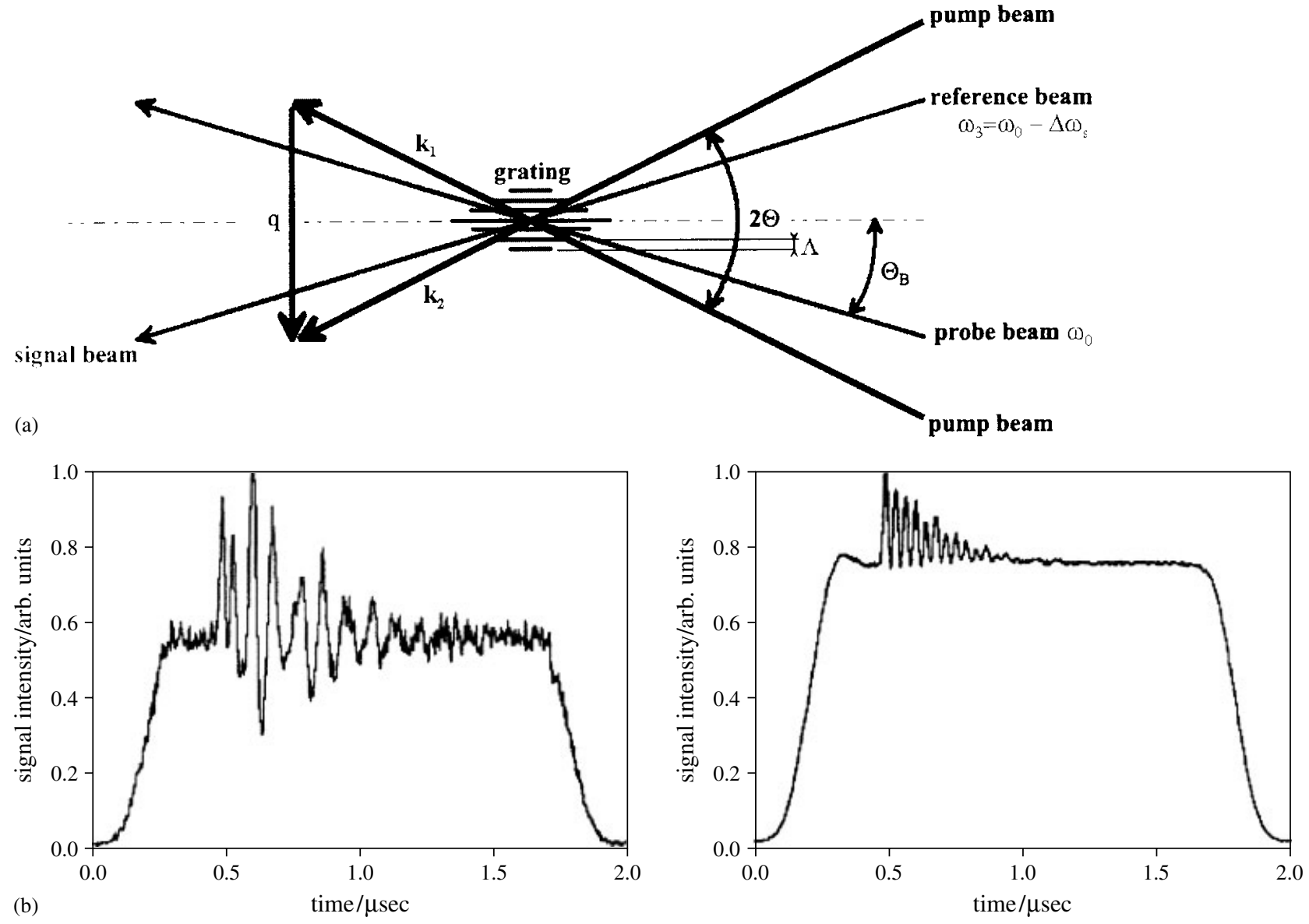

Figure 1. Laser-induced gratings: excitation and heterodyne detection. (a) Principle of standard and heterodyne LIG signal detection schemes; (b) examples of standard (right) and heterodyne (left) single-shot LIG signals obtained in the preliminary laboratory measurements under 'no flow' conditions; the heterodyne LIG signal is seen on the top of the rectangular pulse of the reference beam, the observable modulation resulting from the interference of the signal and the reference beams.

The counter-propagating acoustic waves form a standing wave and thereby a spatially periodic density grating that oscillates in time. The temporal evolution of this grating can be detected by diffracting the beam of a $\mathrm{cw}$ probe laser (with a frequency $\omega_{0}$ ), directed at the Bragg angle $\theta_{\mathrm{B}}$ to the optical axis. The resulting signal beam will be diffracted symmetrically, at the same angle to the optical axis, as shown in Fig. 1(a), and will comprise contributions with Doppler-shifted frequencies $\omega_{1,2}=\omega_{0} \pm q v_{s}$, respectively, that originate from one of the two acoustic waves. Here $\Omega_{\mathrm{a}}=q v_{\mathrm{s}}$ is the frequency of the acoustic wave, determined by the fringe spacing and the sound velocity. Interference of these two signal beam contributions provides an oscillation of the photodetector signal at the frequency $\Omega_{0}=\omega_{1}-\omega_{2}=$ $2 q v_{\mathrm{s}}=2 \Omega_{\mathrm{a}}$ [see Fig. 1(b), left]. The decay of the signal amplitude is determined by the damping of the acoustic wave and the finite transversal dimensions of the probe volume.

If the grating is formed in a flow with velocity $\mathbf{v}_{\mathbf{f}}$, the frequencies of the two signal beam contributions acquire an additional Doppler shift and become $\omega_{1,2}=$ $\omega_{0} \pm q v_{\mathrm{s}}+\mathbf{q v}_{\mathrm{f}}$. The temporal shape and the oscillation frequency of the photodetector signal remain unchanged in this case. However, the flow velocity can be measured by the heterodyne technique. If the signal beam is overlapped with an additional coherent reference beam of a frequency $\omega_{3}$, as shown in Fig. 1(a), the temporal evolution of the heterodyne photodetector signal is varied significantly [see Fig. 1(b), right], and three oscillation frequencies appear in its power spectrum, namely $\Omega_{0}, \Omega_{1}=\Omega_{\mathrm{a}}-\Delta \omega_{\mathrm{s}}-\mathbf{q v}_{\mathrm{f}}$, and $\Omega_{2}=\Omega_{\mathrm{a}}+\Delta \omega_{\mathrm{s}}+\mathbf{q} \mathbf{v}_{\mathrm{f}}$, where $\Delta \omega_{\mathrm{s}}=\omega_{0}-\omega_{3}$, as demonstrated in Fig. 2. The frequency shift $\Delta \omega_{\mathrm{s}}$ and the length of the grating vector $q$ can be obtained from calibration measurements of $\Omega_{1}, \Omega_{2}$ and $\Omega_{0}$ under 'no flow' conditions and known temperature $T_{0}$ and sound velocity $v_{\mathrm{s}}\left(T_{0}\right): \Delta \omega_{\mathrm{s}}=\left(\Omega_{2}-\Omega_{1}\right)\left(T_{0}\right) / 2, q=\Omega_{0}\left(T_{0}\right) / 2 v_{\mathrm{s}}\left(T_{0}\right)$. Thus, in the flow, the measured difference $\Omega_{2}-\Omega_{1}=2 \Delta \omega_{\mathrm{s}}+2 \mathbf{q v}_{\mathrm{f}}$, determined from the power spectrum, allows one to evaluate the component of the flow velocity parallel to $\mathbf{q}, v_{q}$, including the direction of the flow:

$$
v_{q}=\frac{\left(\Omega_{2}-\Omega_{1}\right)-2 \Delta \omega_{\mathrm{s}}}{2 q}=\left[\frac{\left(\Omega_{2}-\Omega_{1}\right)-2 \Delta \omega_{\mathrm{s}}}{\Omega_{0}\left(T_{0}\right)}\right] v_{\mathrm{s}}\left(T_{0}\right)
$$




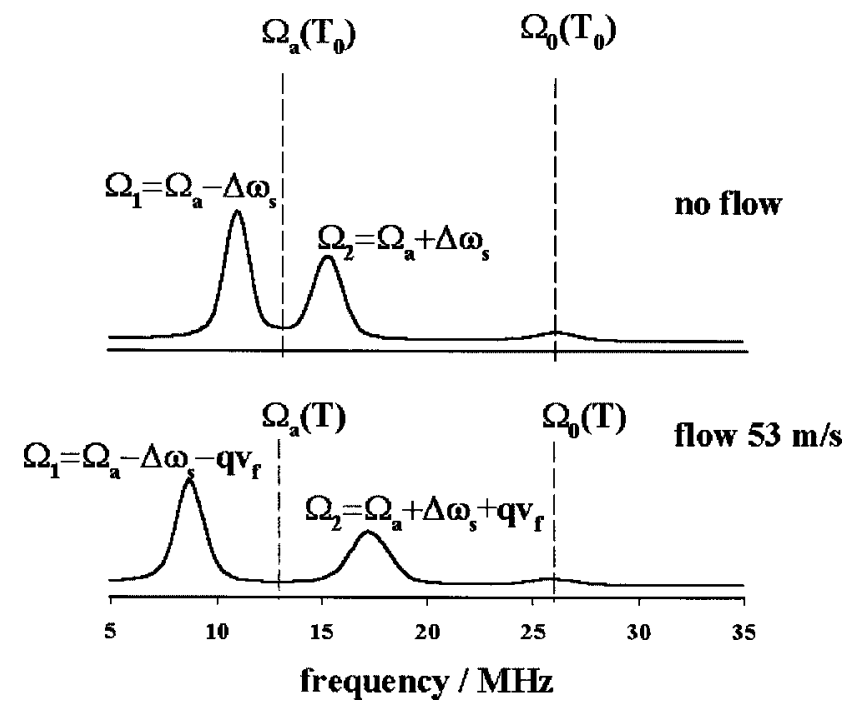

Figure 2. An example of the power spectrum of a heterodyne LIG signal, from quiescent gas at $T_{0}=295 \mathrm{~K}$ ('no flow' calibration conditions) and from a flow. The preliminary laboratory measurements under stationary conditions in a submerged air jet in ambient air at the exit of a slot nozzle (similar to that one employed in Ref. 4). Characteristic values are $\Omega_{\mathrm{a}}\left(T_{0}\right)=13.35 \mathrm{MHz}$ and $\Delta \omega_{\mathrm{s}}=2.2 \mathrm{MHz}$.

In addition, from $\Omega_{1}+\Omega_{2}=\Omega_{0}$, one can determine the sound velocity at the location of measurement: $v_{\mathrm{s}}=\Omega_{0} / 2 q$, and thereby, if the composition of the medium is known, the local temperature. If the composition in the flow is not changing compared with conditions of calibration measurements, the local temperature is determined as

$$
T=\left[\Omega_{0} / \Omega_{0}\left(T_{0}\right)\right]^{2} T_{0}
$$

It should be emphasized that in case of a non-zero shift $\Delta \omega_{\mathrm{s}}$ between the frequencies of the probe and the reference beams being employed, the observed two peaks in the power spectrum of the photodetector signal, at $\Omega_{1}$ and $\Omega_{2}$ (see Fig. 2), exist even if there is no flow. With a flow, the peaks are shifted due to the Doppler effect, as related to their initial positions in quiescent gas: with the increase of the flow velocity in the direction of $\mathbf{q}$ the peaks move apart, whereas if the flow has the opposite direction, the peaks move closer towards each other. Hence both the value and the sign of the local flow velocity component can be determined from the power spectrum in this case, which is essential if a recirculation zone has to be detected.

If the peaks at $\Omega_{1}$ and $\Omega_{2}$ in the power spectrum were infinitely narrow, the flow velocity component $v_{q}$ could be defined in the range given by $-\left|\Delta \omega_{\mathrm{s}}\right| / q<v_{q}<$ $v_{\mathrm{s}}\left(T_{0}\right)-\Delta \omega_{\mathrm{s}} / q$. However, this is not the case since the peaks have a non-zero width owing to the finite lifetime of a LIG. Hence, in practice, the operation range is slightly reduced. Furthermore, a steep increase of the background intensity is usually observed in the zero-frequency region of the power spectrum, which additionally reduces the upper limit of $v_{q}$ values measurable by the technique.

In contrast in the case that the frequency shift $\Delta \omega_{\mathrm{s}}$ is zero (i.e. $\omega_{3}=\omega_{0}$ ), just one peak at $\Omega_{1}=\Omega_{2}=\Omega_{\mathrm{a}}$ exists for quiescent gas, the slow movement of the gas initially resulting only in its broadening. Thus, the power spectrum contains no information on the direction of the flow, while the determination of small values of flow velocities is hindered.

\section{EXPERIMENTAL}

The scheme of the experimental set-up to realize the technique described above is shown in Fig. 3. A repetitivelypulsed Nd:YAG laser (Coherent Infinity 40-100), with $\lambda_{\mathrm{p}}=1064 \mathrm{~nm}$, energy $\approx 100 \mathrm{~mJ}$, repetition rate $100 \mathrm{~Hz}$, pulse length $3.5 \mathrm{~ns}$ and spectral bandwidth $250 \mathrm{MHz}$, provided the two pump beams to create an electrostrictive grating in a standard experimental arrangement., ${ }^{2,4}$

The excitation beams were focused by a lens $(f=$ $1000 \mathrm{~mm}$ ). The crossing angle $2 \theta$ was about $1.68^{\circ}$, which corresponded to $\Lambda \approx 36 \mu \mathrm{m}$. The respective value of the acoustic frequency for $\mathrm{N}_{2}$ at $T=295 \mathrm{~K}$ was $\Omega_{\mathrm{a}} \approx 9.7 \mathrm{MHz}$. The characteristic dimensions of the probe volume were

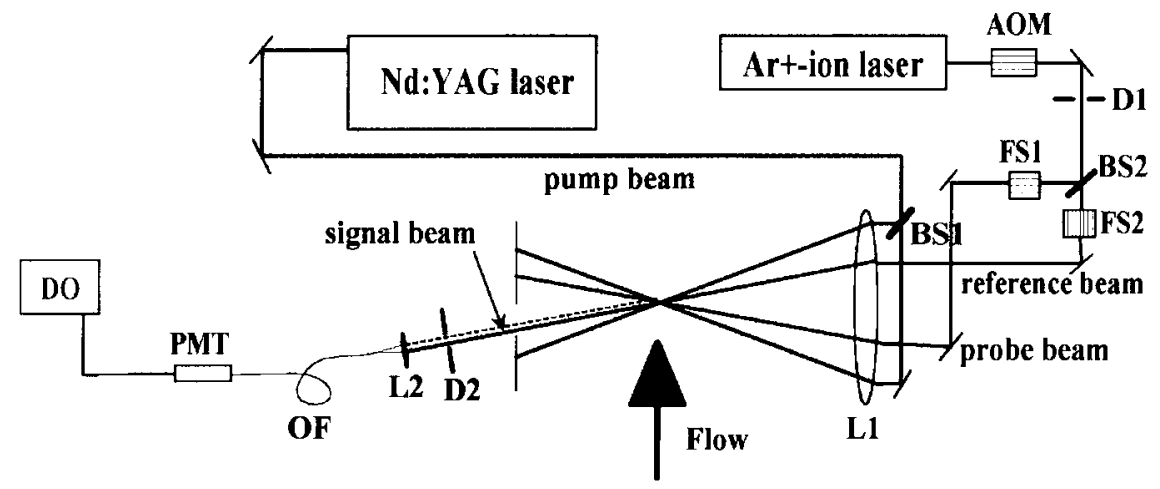

Figure 3. Scheme of the experimental set-up. BS1,2 = beamsplitters; FS1,2 = acousto-optical frequency shifters; L1,2 = focusing lenses; D1,2 = iris diaphragms; OF = optical fiber; PMT = photomultiplier tube; DO = digital oscilloscope; $\mathrm{AOM}=$ acousto-optical modulator of the probe and the reference beams. 
estimated to be about $350 \mu \mathrm{m}$ in diameter and $18 \mathrm{~mm}$ in length. The acoustic decay time and the acoustic transit time for a LIG signal ${ }^{2,4}$ could thus be estimated for these conditions as $\tau_{\mathrm{a}} \approx 2.3 \mu \mathrm{s}$ and $\tau_{\mathrm{tr}} \approx 0.5 \mu \mathrm{s}$, respectively.

The beam of a cw argon ion laser (Coherent Innova90 ), with $\lambda=514.5 \mathrm{~nm}$ and total power $\approx 200 \mathrm{~mW}$, was used to provide both the probe and the reference beams. These beams were focused to the probe volume by the same lens. To obtain phase matching of all the beams, a 2-D forward geometry was used. Two frequency-tunable acousto-optical modulators (Brimrose, TEF 8030-514) were employed as independently driven frequency shifters to introduce a shift $\Delta \omega_{\mathrm{s}}$ between the frequencies of the probe and the reference beams. The modulation frequencies were adjusted by selecting the levels of modulators' drivers d.c. control voltages. The resulting shift was installed in our case as roughly equal to $3.5 \mathrm{MHz}$, which corresponds to the operation range $-100 \leq v_{q} \leq 190 \mathrm{~m} \mathrm{~s}^{-1}$, accounting for the limitations described above. The reference beam, directed symmetrically to the probe beam, overlapped with the signal beam. Both beams were passed through the spatial filter (a diaphragm, a focusing lens and an optical fiber) and were detected by a fast photomultiplier tube (Philips XP1017). To avoid damage to the photomultiplier by the high $\mathrm{cw}$ light level of the reference beam, the third acousto-optical modulator (ISOMET M1205C-2) was employed to switch on the argon ion laser radiation for a short period synchronously with the pump laser shot (gatewidth $\approx 2 \mu \mathrm{s}$ ). Hence the probe and the reference beams were present only during the lifetime of the grating. Temporally resolved acquisition of LIG signals, single-shot or accumulated, was performed using a digital oscilloscope (Tektronix TDS 744A) with a full bandwidth of $500 \mathrm{MHz}$ and a sampling rate up to $2 \mathrm{GHz}$. The digitized signals were directly transferred to a PC.

Measurements were performed at the test site P6.2, dedicated to cold-gas nozzle flow investigations, at the Space Propulsion Institute of the German Aerospace Center at Lampoldshausen. Flows from a subscale thrust optimized, parabolic rocket nozzle (see Fig. 4) were studied. The nozzle had a $20 \mathrm{~mm}$ throat diameter $110 \mathrm{~mm}$ exit diameter and an overall length of $125 \mathrm{~mm}$ from the nozzle throat to the nozzle exit. It was mounted inside a high-altitude simulation chamber, with an attached supersonic diffuser. Nitrogen at ambient temperature $T_{\mathrm{c}}=295 \mathrm{~K}$ and stagnation pressures $p_{\mathrm{c}}$ up to $40 \mathrm{bar}$, increasing either in steps of 5 bar or at a constant rate, was supplied to the nozzle. The measurements were performed in the jets at ambient pressure $p_{\mathrm{a}}=1 \mathrm{bar}$ (the chamber windows open) or at lower pressures (the windows closed).

The $y$,z-plane of the coordinate system was selected to contain the plane of the nozzle throat, and the $x$-axis was set to coincide with the symmetry axis of the nozzle and to point in the direction of the main flow. The laser beams were adjusted to lie within the $x, y$-plane, the $\mathbf{q}$ vector being directed along the $x$-axis, so that the probe

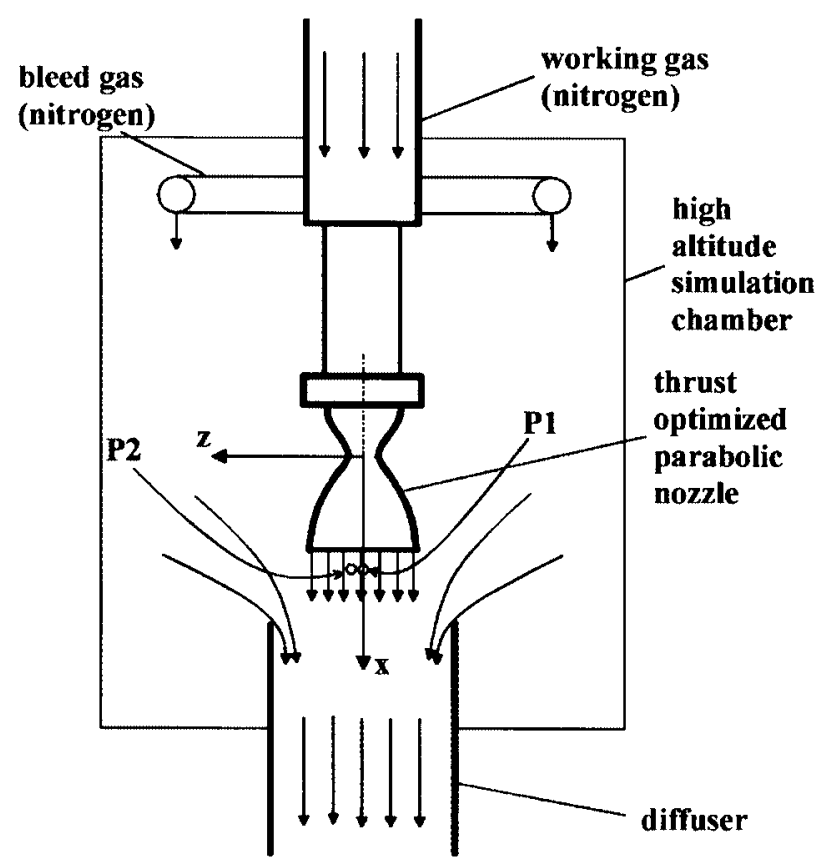

Figure 4. Schematic of the cold-gas test bench P6.2 with a subscale thrust-optimized parabolic rocket nozzle mounted inside a high-altitude simulation chamber. Measurement points (P1 and P2) are indicated.

volume extended along the $y$-axis. Preliminarily, only two measurement locations inside the flow, on- and off-axis and $50 \mathrm{~mm}$ downstream from the exit plane of the nozzle, were selected, in accordance with CFD calculations, namely P1 $(175,0,0 \mathrm{~mm})$ and P2 $(175,0,15 \mathrm{~mm})$. It should be noted that the longitudinal spatial resolution of the technique along the $y$-axis (the length of the probe volume) and the off-axis displacement of the measurement location were consistent with the expected transversal dimensions of the recirculation zone (characteristic diameter in the $y, z$-plane of $\sim 60 \mathrm{~mm}$ at $\left.p_{\mathrm{c}} / p_{\mathrm{a}}=10\right)$, as given by CFD calculations. ${ }^{8}$

\section{RESULTS AND DISCUSSION}

The calibration measurements in quiescent ambient air provided the difference $\Delta \omega_{\mathrm{s}}=3.40 \pm 0.05 \mathrm{MHz}$ between the frequencies of the probe and the reference beams, which corresponds to the expected value of $\sim 3.5 \mathrm{MHz}$, and the value $\Omega_{0}\left(T_{0}\right)=19.40 \pm 0.05 \mathrm{MHz}$ to determine the length of the grating vector $q$. Prior to starting the main flow through the nozzle, the chamber was slowly flooded with nitrogen. The single-shot velocity measurements performed during this period exhibit a Gaussian shaped distribution with a small mean flow velocity $v_{q}=1.5 \pm 0.3 \mathrm{~m} \mathrm{~s}^{-1}$ and a standard deviation of $2.7 \pm 0.2 \mathrm{~m} \mathrm{~s}^{-1}$. This value of $2.7 \mathrm{~m} \mathrm{~s}^{-1}$ can be regarded as an estimate of the limiting precision of a single-shot velocity measurement achievable using the realized technique. It is worth noting that this estimate is in accordance with the error of frequency difference 
determination in those power spectra that were obtained as a result of the calibration measurements.

During the measurements at location P1, performed at ambient pressure $p_{\mathrm{a}}=1 \mathrm{bar}$, with the chamber windows open, the stagnation pressure $p_{c}$, after being increased in steps of 5 bar, was then kept constant for about $15 \mathrm{~s}$, while about 1000 single-shot measurements were acquired. Almost in the entire range of stagnation pressures a strong and pulseto-pulse fluctuating background dominated the temporal evolution of grating signals, while the signal power spectra exhibited peaks that could not be definitely assigned to the expected frequencies. Only at $p_{\mathrm{c}}=10$ bar was the signal quality reasonably good and flow velocity measurements could be performed. The examples of averaged LIG signals, that provide information on local gas temperature, are given for two different stagnation pressures in Fig. 5 and illustrate the above-mentioned deterioration of signal quality at a given measurement location at pressures other than 10 bar.

Figure 6 depicts an example of the power spectrum of a single-shot LIG signal measured at 10 bar. The constraint
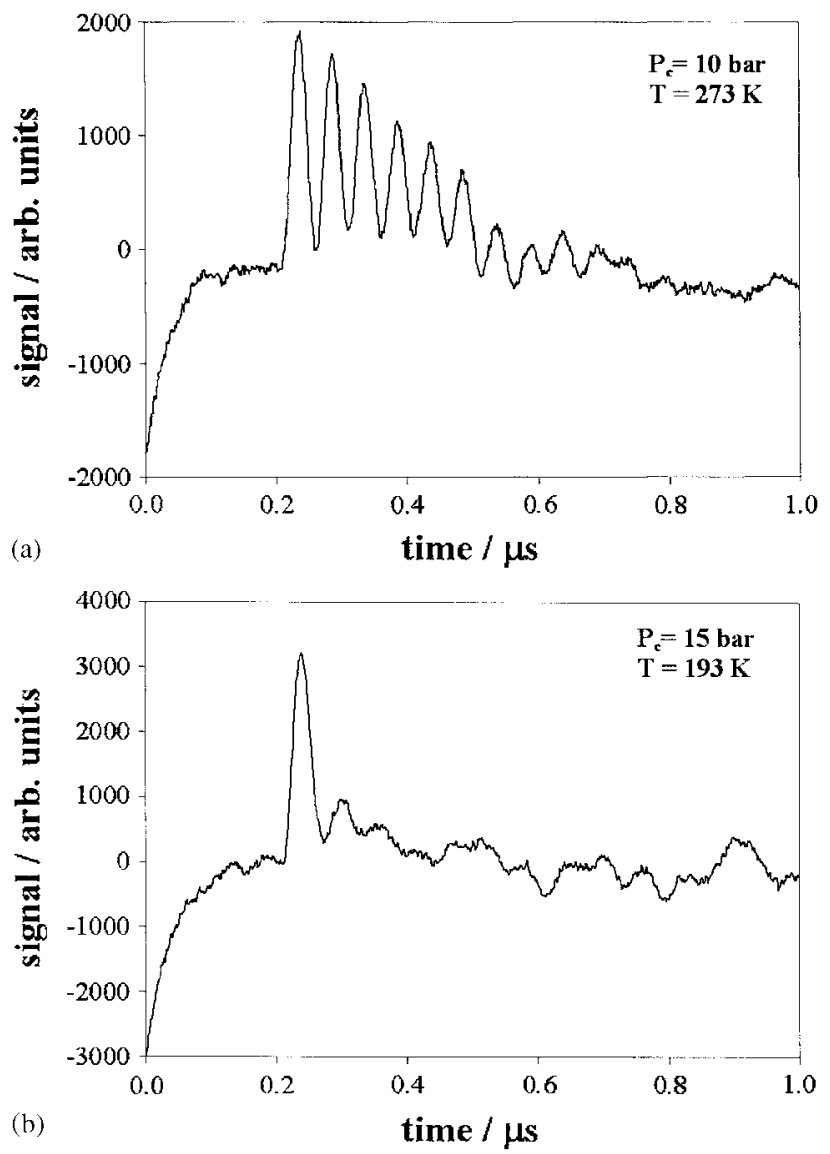

Figure 5. Measurement at point P1 (on-axis): two averaged signals at different stagnation pressures and local flow temperatures: (a) $p_{\mathrm{c}}=10 \mathrm{bar}, T=273 \mathrm{~K}$, averaging over 1000 shots; reasonably good signal quality; (b) $p_{\mathrm{c}}=15$ bar, $T=193 \mathrm{~K}$, averaging over 2000 shots; poor signal quality.

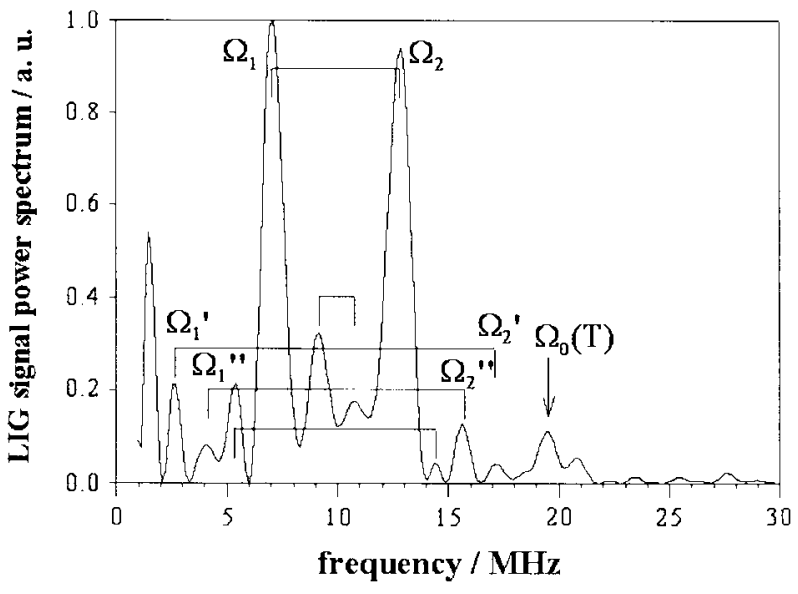

Figure 6. Example of a LIG signal power spectrum for a single-shot signal at $p_{\mathrm{c}}=10$ bar.

on the selection of pairs of peaks, that allows one to determine the flow velocity, is that their frequencies obey the relation $\Omega_{1}+\Omega_{2}=\Omega_{0}(T)$, with the measured value of local temperature $T \approx 273 \mathrm{~K}$ [see Fig. 5(a)] being in reasonable agreement with CFD calculations. The two most prominent lines of the power spectrum are assigned to the frequencies $\Omega_{1}$ and $\Omega_{2}$, that correspond to a flow velocity $v_{q}=-17.4 \mathrm{~m} \mathrm{~s}^{-1}$, which is considered to be dominant inside the probe volume and taken as a measured value. However, one can see that other peaks, with smaller amplitudes, are also present and, as indicated in Fig. 6, other combinations of these peaks ( $\Omega_{1}^{\prime}$ and $\Omega_{2}^{\prime}, \Omega_{1}^{\prime \prime}$ and $\Omega_{2}^{\prime \prime}$, etc.), that satisfy the constraint implied, can be found. A smaller frequency difference between the two peaks corresponds to a smaller velocity and vice versa. Thus, velocities between -87.6 and $131.0 \mathrm{~m} \mathrm{~s}^{-1}$ can be obtained. A possible explanation of this observation could be the following: the flow pattern in the vicinity of the probe volume is inhomogeneous in the $y$ direction, across the main flow, so that a LIG consists of a few segments of a finite length along the probe volume, and these segments move with different velocities along the $x$-axis. The amplitudes of peaks in the power spectrum can be roughly regarded as proportional to the lengths of the grating segments within the corresponding flow jets (i.e. thickness of these jets). Hence, only a pair of the largest peaks is selected for a given single-shot signal to provide a value of flow velocity at a given $x$-position.

The results of single-shot velocity measurements were compiled into normalized histograms, which were fitted by a normal (Gaussian) distribution, as shown in Fig. 7. Figure 7(a) illustrates the initial quasi-'no flow' measurements, with the chamber being slowly flooded with nitrogen. Figure 7(b) presents the data obtained at location P1 and fitted by a normal distribution with correlation coefficient $>0.97$, the mean velocity of the distribution equal to $v_{q}=-4.5 \pm 0.5 \mathrm{~m} \mathrm{~s}^{-1}$ and the standard deviation estimated 

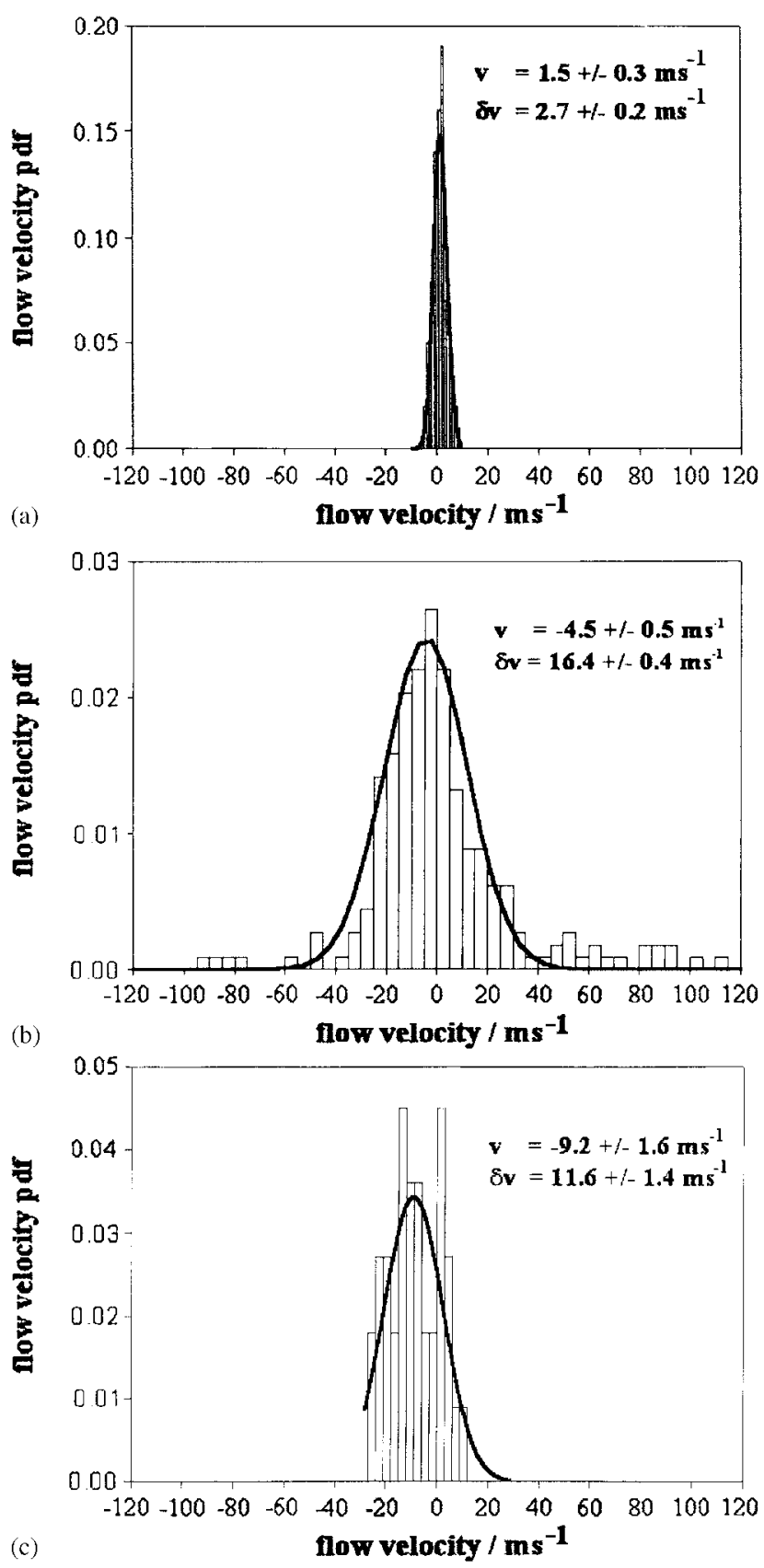

Figure 7. Velocity histograms at two measurement points $\mathrm{P} 1$ (on-axis) and P2 (off-axis), under different conditions of stagnation pressure variation; fitted Gaussian distribution is shown by the solid line: (a) Point P1, quasi 'no flow' conditions, the chamber being slowly flooded with nitrogen; (b) point $\mathrm{P} 1$, $p_{\mathrm{c}}=10$ bar; (c) point P2, $p_{\mathrm{c}}=10$ bar.

to be $16.4 \pm 0.4 \mathrm{~m} \mathrm{~s}^{-1}$. The low absolute values of the measured velocities $v_{q}$, together with the clear evidence of a local flow component with a direction opposite to the main flow, indicate the existence of a recirculation zone at a given location and stagnation pressure. The larger width of the distribution, as compared with that shown in Fig. 7(a), can possibly be ascribed to the significant temporal fluctuations of the flow pattern and both the position and dimensions of the recirculation zone at a given stagnation pressure, detected from shot to shot.

During the measurements at location P2 (off-axis), performed at reduced ambient pressures, with the chamber windows closed, the stagnation pressure $p_{\mathrm{c}}$ was increased up to 40 bar at a constant rate of $1 \mathrm{bar} \mathrm{s}^{-1}$. Again, only within a range of about 1 bar width, centered at $p_{\mathrm{c}} \approx$ 10 bar, were velocity measurements possible. A mean velocity $v_{q}=-9.2 \pm 1.6 \mathrm{~m} \mathrm{~s}^{-1}$ and a standard deviation of $11.6 \pm 1.4 \mathrm{~m} \mathrm{~s}^{-1}$ were determined [see Fig. 7(c)]. Outside this pressure range the measured signals were dominated by a strong fluctuating background that rendered velocity measurements impossible. This background may be caused by scattering of the probe beam on turbulences, that increase with the growth of flow velocities, attendant on a displacement of a recirculation zone along the flow or its disappearance as pressure $p_{\mathrm{c}}$ increases.

Variation of velocities $v_{q}$ along the nozzle axis, given by CFD calculations for stagnation pressures of 8 and 10 bar, are depicted in Fig. 8. A striking qualitative difference between the two distributions can be noted. Negative velocities at $p_{\mathrm{c}}=10 \mathrm{bar}$ indicate the existence of a recirculation zone, with a length of about $80 \mathrm{~mm}$, the position of the measurement point P1 being inside this zone. The results presented in Fig. 8 allow also one to assume that the dependence of both flow velocities pattern and the recirculation zone dimensions (boundaries) on $p_{c}$ is fairly strong at pressures around 10 bar. Our measurements strongly support these calculations in two respects: first, we measured a negative velocity component at location $\mathrm{P} 1$, and second, the recirculation zone was observed the chosen location only in a relatively narrow pressure range. Although the values of measured $\left(-4.5 \mathrm{~m} \mathrm{~s}^{-1}\right)$ and calculated $\left(-30 \mathrm{~m} \mathrm{~s}^{-1}\right)$ velocities are noticeably different, it is worth mentioning that the direct comparison of these two values should be critically assessed taking into account the accuracy

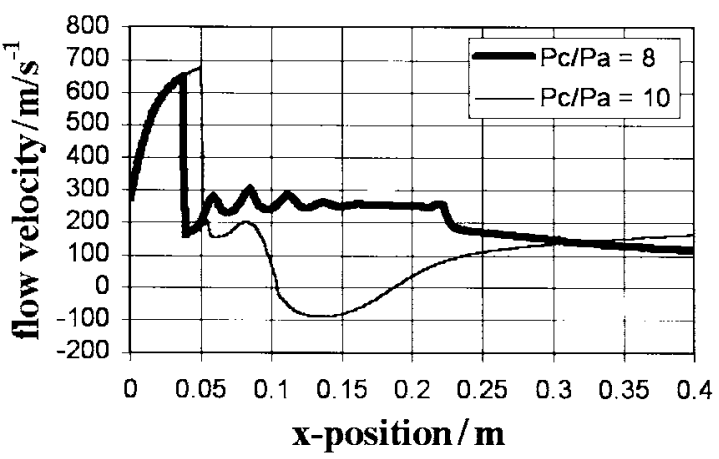

Figure 8. CFD calculations of the variation of flow velocity component $v_{q}$ along the nozzle axis for $p_{\mathrm{c}}=8$ bar (solid thick line) and $p_{\mathrm{c}}=10$ bar (solid thin line); the position of the measurement point P1 is indicated by a vertical line at $0.175 \mathrm{~m}$. 
of the pressure measurements during the experiment. In addition, the inability to observe the recirculation zone at a given measurement location at stagnation pressures above 10 bar correlates with CFD calculations, that predict the displacement of the recirculation zone further downstream at higher pressures.

The appearance of a recirculation zone is associated with the reattachment of the separated flow to the nozzle wall, inducing lateral forces to the walls of a nozzle. Our measurements also agree with earlier side-load measurements, performed at the DLR test site independently, ${ }^{8}$ as far as they explain the appearance of the pronounced peak of the side-load observed at $p_{\mathrm{c}} \approx 10 \mathrm{bar}$.

\section{CONCLUSIONS}

For the first time, the laser-induced gratings technique has been applied to investigate a real fluid-dynamic problem. Flow velocity measurements, aimed at validating CFD calculations, were performed inside the cold flow of nitrogen from a sub-scaled thrust optimized parabolic rocket nozzle at stagnation pressures up to $40 \mathrm{bar}$. The results clearly prove the existence of a recirculation zone in the flow field of the plume outside the nozzle near its axis, that agrees well with predictions of CFD calculations, and should stimulate further detailed theoretical investigations.

\section{Acknowledgements}

The authors are grateful to P. Radi for assistance in writing the data acquisition software. The authors are also indebted to A. Wokaun for continuous support of this work. Financial support by the Swiss Federal Department of Energy (BFE), LEC ERCOFTAC, Switzerland, and Astrium GmbH, Germany, is gratefully acknowledged.

\section{REFERENCES}

1. Walker DJW, Williams RB, Ewart P. Opt. Lett. 1998; 23: 1316.

2. Kozlov DN, Hemmerling B, Stampanoni-Panariello A. Appl. Phys. B 2000; 71: 585 .

3. Schlamp S, Cummings EB, Sobota TH. Opt. Lett. 2000; 25: 224.

4. Hemmerling B, Kozlov DN, Stampanoni-Panariello A. Opt. Lett. 2000; 25: 1340.

5. Schlamp S, Allen-Bradley E. AIAA Paper (American Institute of Aeronautics and Astronautics) 2000-0376, Reno, Nevada, USA 2000.

6. Hart RC, Balla RJ, Herring GC. Appl. Opt. 2001; 40: 965.

7. Frey M, Hagemann G. AIAA Los Angeles, CA, USA, Paper 9928151999.

8. Kwan W, Stark R. AIAA Indianopolis, IN, USA, Paper 2002-2864 2002. 